\title{
Le Moyen Âge vu d'ailleurs II
}

São Paulo, 27-31 octobre 2003

Lênia Márcia Mongelli

\section{(2) OpenEdition}

Journals

Édition électronique

URL : https://journals.openedition.org/cem/923

DOI : $10.4000 /$ cem.923

ISSN : 1954-3093

Éditeur

Centre d'études médiévales Saint-Germain d'Auxerre

Édition imprimée

Date de publication : 15 août 2004

ISSN : 1623-5770

Référence électronique

Lênia Márcia Mongelli, « Le Moyen Âge vu d'ailleurs II », Bulletin du centre d'études médiévales d'Auxerre I BUCEMA [En ligne], 8 | 2004, mis en ligne le 20 décembre 2006, consulté le 22 septembre 2022. URL http://journals.openedition.org/cem/923 ; DOI : https://doi.org/10.4000/cem.923

Ce document a été généré automatiquement le 22 septembre 2022.

\section{(c) (1) (2)}

Creative Commons - Attribution - Pas d'Utilisation Commerciale - Partage dans les Mêmes Conditions 4.0 International - CC BY-NC-SA 4.0

https://creativecommons.org/licenses/by-nc-sa/4.0/ 


\title{
Le Moyen Âge vu d'ailleurs II
}

São Paulo, 27-31 octobre 2003

\author{
Lênia Márcia Mongelli
}

Du 27 au 31 octobre 2003, l'Institut d'Études Avancées (Instituto de Estudos Avançados. IEA) de l'Université de São Paulo (USP/Brésil) a reçu, pour la première fois, et à l'initiative du Centre d'études médiévales d'Auxerre, un groupe de médiévistes français, espagnols, portugais, argentins et brésiliens, réunis pour discuter de questions d'historiographie générale et des tendances actuelles de la médiévistique. Cette rencontre de 24 chercheurs, ayant des intérêts communs mais avec de rares occasions de se rassembler, comptait un public restreint de professionnels du secteur, de doctorants et de post-doctorants en histoire médiévale. Seize communications ont été présentées lors de huit tables rondes différentes (deux d'“historiographie" et six sur les "recherches récentes"), coordonnées par huit professeurs parmi les assistants. Le nombre réduit d'intervenants et de public a donné de l'identité à la rencontre, qui s'est définie comme un "atelier d'études et de formation à la recherche", où l'échange d'idées et l'examen de travaux individuels étaient possible.

2 La réalisation effective de ces objectifs est à créditer à la coordination de la rencontre, sous la responsabilité générale d'Eliana Magnani (CNRs/Auxerre) et, en ce qui concerne l'organisation brésilienne, d'Hilário Franco Júnior (USP) et de Flávio de Campos (USP). L'ambiance agréable et intense dans laquelle se sont déroulés ces quatre jours est dûe à la bonne distribution du temps. Chaque communication de 45 minutes était suivie de 30 minutes réservées aux débats passionnants et instructifs. Il y a eu des moments. et ils n'étaient pas des moindres. où les problèmes abordés à la suite de la communication ont considérablement enrichi le sujet de départ, dans un foisonnement de nouveaux thèmes, d'approches plus justes, de méthodologies interdisciplinaires, de différences de points de vue, dans une sorte de cours d'historiographie(s). Il faut encore souligner la collaboration administrative décisive de l'IEA, dont le personnel a prodigué des efforts notables pour que tout se déroule à souhait.

3 La dynamique de l'atelier avait été précédemment testée : un premier atelier avait eu lieu à Auxerre, en octobre 2002, où les chercheurs latino-américains avaient présenté leurs travaux aux collègues français ${ }^{1}$; le deuxième s'est tenu au Brésil, où c'était au tour des 
français, des argentins, des espagnols et des portugais de se présenter. Ces deux dernières nationalités ont rejoint celles du départ et étaient représentées par Pascual Martínez Sopena (Université de Valladolid) et par Judite A. Gonçalves de Freitas (Université Fernando Pessoa. Porto). Les deux intervenants ont fait connaître "les traditions et les tendances" de la médiévistique dans leurs pays ${ }^{2}$, tout comme cela s'était produit à Auxerre. Le dernier jour, deux brésiliens sont aussi intervenus, faisant les honneurs de la maison.

D'une rencontre à l'autre, plus que la diversification thématique (plusieurs recherches, d'hier et d'aujourd'hui, continuent de suivre leur cours), plus que la variété des sujets, on a cherché à réfléchir sur la formulation de problèmes et les nouveautés méthodologiques offertes par l'anthropologie, l'archéologie, la linguistique et l'histoire de l'art. Comme l'a montré l'équipe française, par la voix de Jean-Claude Schmitt, dont le commentaire principal a tourné autour de l'œuvre Tendances actuelles de l'Histoire du Moyen Âge en France et en Allemagne (Paris, Publications de la Sorbonne, 2003) ${ }^{3}$, l'idée vient de loin, plus précisément d'un colloque tenu à Sèvres en 1997, puis d'un autre à Göttingen, en 1998, lorsque l'on a insisté, à ces deux occasions, sur la nécessité périodique "d'historiciser l'Histoire", de revoir les progrès et les changements du traitement des sources documentaires, largement étendues par des consortium multidisciplinaires.

5 Comme on peut l'observer, les ateliers I (Auxerre) et II (São Paulo) ont été conçus en bloc (avec des perspectives pour le III, le IV, etc.) et prétendaient, dès le départ, se circonscrire à trois grands thèmes : l'historiographie à partir de l'après-guerre ; l'histoire et l'anthropologie ; l'histoire sociale et économique ${ }^{4}$. Cependant, les séminaires se sont déroulés d'une façon naturellement souple, privilégiant certains aspects. par exemple, l'iconographie, la liturgie et le Droit, en reléguant d'autres, comme l'histoire économique, sur laquelle l'attention avait été attirée à Göttingen. Quant à la chronologie, la périodisation était bien équilibrée entre le haut, le bas et le Moyen Âge central, présentant une vision d'ensemble du panorama multiforme du Moyen Âge.

6 Les débats après les présentations ont fini par constituer le point culminant de la rencontre, du moins pour avoir sélectionné de complexes questions ponctuelles très utiles aux jeunes chercheurs présents, presque tous des latino-américains travaillant loin des sources manuscrites européennes et avec des limitations bibliographiques de plusieurs sortes (Joseph Morsel, dans la synthèse faite à l'atelier d'Auxerre, a montré quelques résultats indésirables et dangereux de ces obstacles). Il serait impossible ici de reproduire la richesse des contributions ; cependant quelques points, repris à chaque communication, en ressortent :

7 - sur la précision conceptuelle : il faut être attentif à la fluidité des mots et à leur polysémie de sens, selon le moment historique. La contextualisation du document commence par la valeur sémiotique du signe dont il se sert ;

8 - si l'intérêt essentiel de l'historien est de comprendre la transformation de la société, des supports méthodologiques, comme ceux qui sont offerts par l'anthropologie culturelle et sociale, doivent l'amener à une procédure essentiellement comparatiste : tout objet se rapportera à d'autres qui lui sont proches dans le temps et l'espace, aussi bien qu'à ceux qui appartiennent à des communautés et groupements plus éloignés, sous peine de faire une micro-histoire isolée ;

9 - l'œuvre. textuelle, iconographique, picturale, musicale, architectonique, etc.. s'offre à un regard qui l'interprète et qui, pour cette même raison, garde une parcelle de 
subjectivité. Constatation qui rapproche les sciences humaines entre elles et place les arts sur des paliers équivalents par le surpoids de l'abstraction. Elle exige du chercheur une rigueur d'évaluation et des outils appropriés, pour que la reconstitution de l'objet soit la plus fidèle à l'espace-temps qui l'a conçu et lui a donné forme.

Des raisonnements fondamentaux, sous l'effet desquels on a développé un nouveau regard. plus critique et exigeant. sur les recherches présentées à São Paulo, dans l'attendu exercice de la réflexion. Nous gardons des leçons pour les futurs ateliers : la plus importante est que les rencontres se suivent étroitement, dans une estimation d'ensemble, pour continuer l'ouverture à la diversification thématique et à l'effervescence des nouveautés historiographiques des deux côtés de l'Atlantique, dans un échange bénéfique aux latino-américains et aux européens, même si ce sont pour des raisons diverses.

11 Voici l'intégralité des contributions présentées :

- Tradiciones y tendencias en el medievalismo español, par Pascual MARTínez sopena (Univ. de Valladolid)

- Os caminhos da história do poder no medievismo português, par Judite A. GONÇALVES DE FREITAS (Univ. Fernando Pessoa. Porto)

- Literatura profética y poder en Byzancio durante los siglos VIII al X, par Pablo UBIERNA (Univ. de Buenos Aires)

- Discurso, gestos y comunicación en la liturgia visigoda (598-711) , par Eleonora DELL'ELICINE (Univ. de Buenos Aires / Univ. Nacional de General Sarmiento)

- Histoire de l'art et iconographie, par Daniel Russo (Univ. de Bourgogne)

- Os pilares do claustro de Moissac, par Maria Cristina PEREIRA (Univ. Federal do Espírito Santo)

- Problèmes de l'image médiévale pour l'historien médiéviste, par Jean-Claude SCHMITT (EHESS)

- La prueba judicial en Castilla durante el siglo XIII, par Marta MADERO (Univ. de Buenos Aires / Univ. Nac. de General Sarmiento)

- Entre memoria y olvido. El tiempo de la costumbre en el derecho medieval, par Paola MICELI (Univ. Nacional de General Sarmiento)

- Do evergetismo ao dom "pro anima” (século IV ao VIII), par Eliana MAGNANI (CNRS. Auxerre/ Dijon)

- Un projet d'enquête : reprendre l'étude de la crise de 1300 dans un cadre méditerranéen, par Monique BOURIN (Univ. de Paris I)

- Les logiques communautaires entre logiques spatiales et logiques catégorielles $\left(\mathrm{XII}^{\mathrm{e}}-\mathrm{XV}^{\mathrm{e}}\right.$ s.), par Joseph MORSEL (Univ. de Paris I)

- Architecture d'intérieur. Édification personnelle et construction ecclésiale, par Dominique IOGNA-PRAT (CNRS - Auxerre/Dijon)

- Pecado e indivíduo en el marco de una antropología cristiana medieval, par Alejandro MORÍN (Univ. de Buenos Aires)

- A agonia lúdica : guerra, competição e fortuna nos jogos medievais, par Flávio DE CAMPOS (Univ. de São Paulo)

- Os sinais da infâmia e o vestuário mouro em Portugal nos séculos XIV e XV, par José Rivair MACEDO (Univ. Federal do Rio Grande do Sul)

Hilário FRANCO JÚNIOR (Univ. de São Paulo), João GOMES (Univ. Estadual Paulista - Franca/SP), Lênia Márcia MONGELLI (Univ. de São Paulo), Marcelo CÂNDIDO DA SILVA (Univ. de São Paulo), María Inés CARZolio (Univ. Nacional de Rosario), Mário Jorge DA MOTTA BASTOS (Univ. Federal 
Fluminense), Néri DE BARROS ALMEIDA (Unicamp - Campinas/SP), Vívian COUTINHO DE ALMEIDA (Univ. de São Paulo).

\section{NOTES}

1.Voir la synthèse de cette rencontre par J. MORSEL dans Études \& Travaux, 7, 2002-2003, p. 93-98.

2.Voir les contributions de P. MARTÍNEZ SOPENA et de J. A. GONÇALVES DE FREITAS ci-après dans ce volume.

3.Voir la contribution de J.-Cl. schMiTT ci-après dans ce volume.

4.Études \& Travaux, 6, 2001-2002, p. 156-162. 